\title{
LAUNCH VEHICLES OF ISAS
}

\author{
YASUO TANAKA \\ Institute of Space and Astronautical Science \\ 3-1-1 Yoshinodai, Sagamihara, Kanagawa-ken 229, Japan
}

\begin{abstract}
The Institute of Space and Astronautical Science (ISAS) has developed a series of launch vehicles for delivering modest scale scientific satellites into space. This capability is unique for an inter-university research institute. The present ISAS launch vehicle, M-3S II, carries a $770 \mathrm{~kg}$ payload into a low earth orbit (LEO). A new program for the development of a more capable vehicle, $M-V$, started recently, which will have a LEO capability three times that of M-3S II. The enhanced launch capability will further expand the scope of the ISAS missions to include planetary explorations.
\end{abstract}

\section{Introduction}

Before describing the launch vehicles of ISAS, it may be useful to describe the two organizations responsible for all space activities of Japan. One is the ISAS operating under the Ministry of Education, Science and Culture. ISAS is responsible for implementing all aspects of space science research programs. The other organization is National Space Developing Agency (NASDA) under the Science and Technolgy Agency. NASDA is responsible for all applications space programs. The individual programs of both space organizations are reviewed and approved by the Space Activities Commission belonging to the Prime Minister's Office from the point of view of complementarity and coherence.

ISAS is a national inter-university research institute, serving the whole of the space science community in Japan. The role of ISAS is the coordination, planning and implementation of the space research programs for the university scientists throughout the country with their direct involvement.

While ISAS is small in scale (total $\sim 300$ of which $\sim 100$ are academic staff), it has some unique features for a research institute. ISAS has developed and possesses its own satellite launch vehicles and launch capability. This feature, in addition to the in-house capabilities of project management, design, tests, tracking and data processing, allows us to conduct all aspects of planning and implementation of scientific missions. ISAS has so far launched nineteen satellites in the past twenty years. Though ISAS missions are all modest in scale, the frequent mission opportunities are enormously important. They ensure continuity of investigations and steady development in each space science discipline. Furthermore, frequent opportunities also allow active engagement of young scientists.

\section{Launch Vehicles}

In order to avoid duplicate efforts by the two space organizations, the government has accorded ISAS and NASDA complementary roles. ISAS developes launch vehicles consisting of solid propellant motors limited until recently to a booster diameter

Y. Kondo (ed.), Observatories in Earth Orbit and Beyond, 339-342.

(C) 1990 Kluwer Academic Publishers. Printed in The Netherlands. 
TABLE I

Basic parameters of the Japanese launch vehicles

\begin{tabular}{|c|c|c|c|c|c|}
\hline \multicolumn{2}{|l|}{ Vehicle } & M-3S II & $M-V$ & H-I & H-II \\
\hline \multirow{3}{*}{\multicolumn{2}{|c|}{$\begin{array}{l}\text { Organization } \\
\text { Propellant }\end{array}$}} & ISAS & ISAS & NASDA & NASDA \\
\hline & & all solid & all solid & liquid & $\mathrm{LH}_{2} / \mathrm{LOX}$ \\
\hline & & 3-stage & 3-stage & $\mathrm{LH}_{2}$ 2nd stage & 2-stage \\
\hline \multicolumn{2}{|c|}{ Total Weight (tons) } & 62 & 128 & 140 & 260 \\
\hline \multirow[t]{2}{*}{ Payload (tons) } & LEO & 0.77 & $\sim 2$ & 3.0 & 10.0 \\
\hline & GEO & & & 0.55 & 2.2 \\
\hline \multirow{2}{*}{\multicolumn{2}{|c|}{$\begin{array}{l}\text { Nose Fairing (m dia.) } \\
\text { Operational Status }\end{array}$}} & 1.6 & 2.5 & 2.44 & 4.0 (or 5.0$)$ \\
\hline & & current & $1995 \sim$ & current & $1993 \sim$ \\
\hline
\end{tabular}

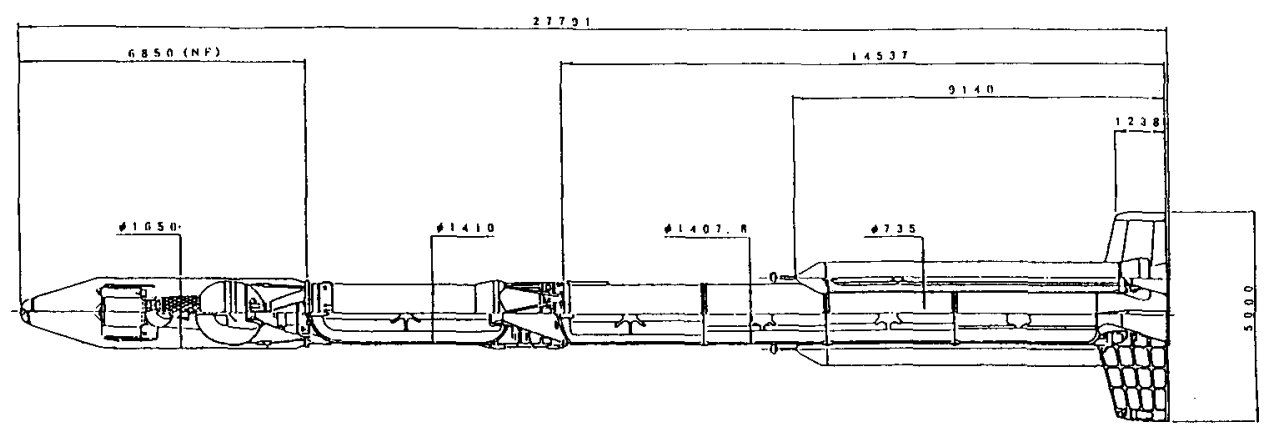

Fig. 1. Schematic diagram of the M-3S II.

of $1.4 \mathrm{~m}$. On the other hand, NASDA is in charge of developing bigger, liquid fuel motors.

ISAS has been steadily improving the capability of its launch vehicle which we call Mu-rocket within the $1.4 \mathrm{~m}$ diameter limitation. The current ISAS launch vehicle is named M-3S II and is the latest version of Mu-rocket, which is a threestage solid propellant motor assisted by two strapped-on boosters. Figure 1 shows a schematic diagram of the M-3S II. This vehicle can launch a $770 \mathrm{~kg}$ payload into a low earth orbit (LEO). Also the M-3S II launchers sent two missions, Sakigake and Suisei, to Comet Halley in 1985; these were the first interplanetary missions for ISAS. The X-ray astronomy observatory Ginga, and the auroral mission Akebono were also launched by these vehicles. The M-3S II launchers will bring two more missions, Solar-A for the next solar maximum and Astro-D an X-ray astronomy observatory each weighing about $420 \mathrm{~kg}$, into near earth $(500 \mathrm{~km}$ circular) orbits in 1991 and 1993, respectively.

Further enhancement of the ISAS launch capability has been strongly desired 


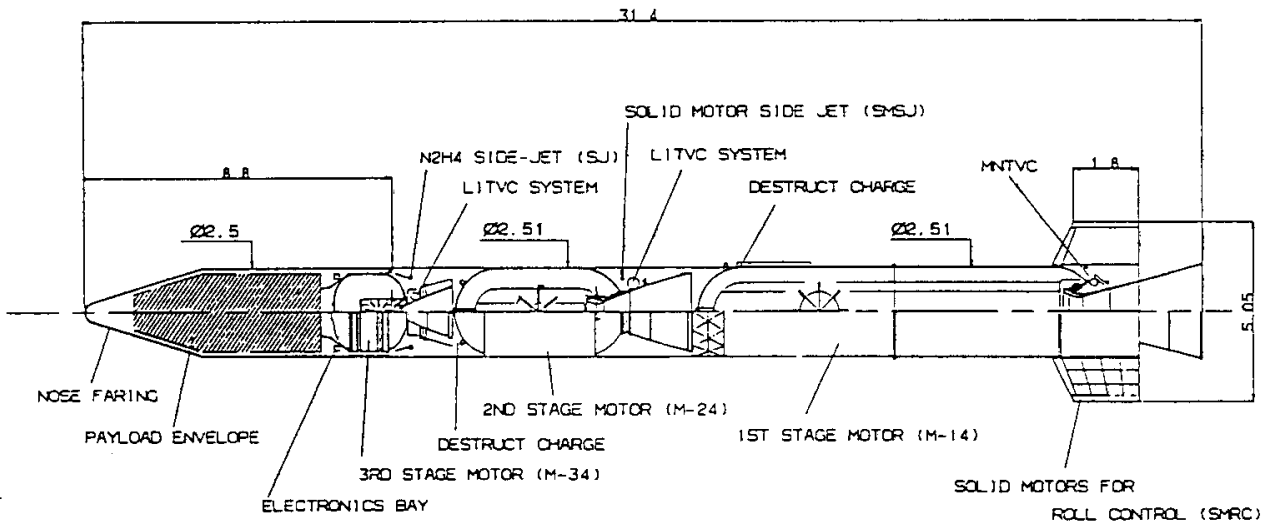

Fig. 2. The configuration of the $M-V$ vehicle.

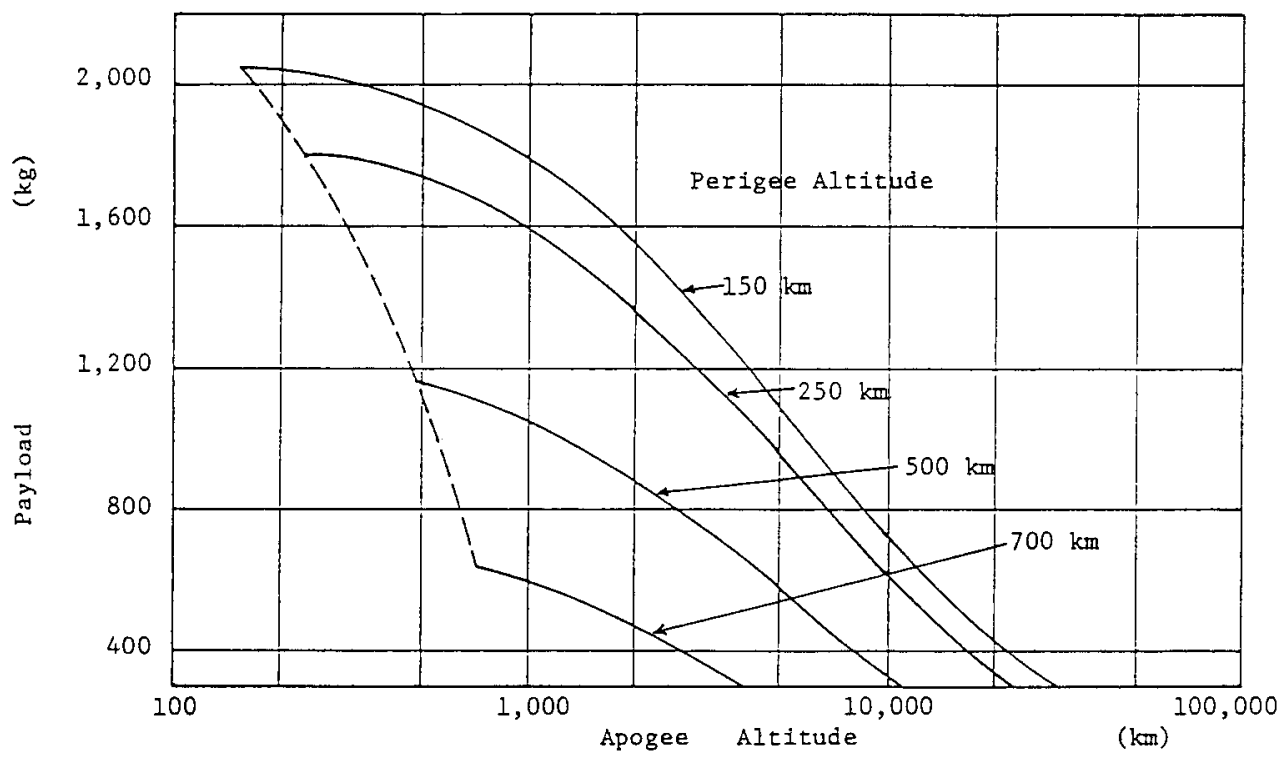

Fig. 3. Payload capability of the $M-V$ vehicle. 
by the scientific community in order to fulfill demands in the coming decade for conducting planetary explorations as well as delivering larger and more sophisticated satellites into near earth orbit. Recently, a new ISAS program for further development of solid-propellant launch vehicles has been approved.

The new launch vehicle named $M-V$ will have a capability about three times that of the M-3S II. The present design configuration of the M-V vehicle is shown schematically in Fig. 2. The launcher consists of three stages of solid propellant motors with an outer diameter of 2.5 meters. As shown in Fig. 3, the M-V vehicle will be capable of launching a payload of $\sim 2$ tons into LEO, or carrying roughly 500 $\mathrm{kg}$ to Venus or Mars with an aid of a kick motor. The M-V development program started this year, and will launch MUSES-B, the first space VLBI mission (VSOP), in early 1995. The basic parameters of the ISAS launch vehicles, M-3S II and M-V, are summarized in Table $\mathbf{I}$.

For the above range of payload mass, the solid propellant vehicles are superior to the liquid-fuel vehicles for modest scale satellites and provide low cost and reliable delivery to orbit. In the design of the $\mathrm{M}-\mathrm{V}$ vehicle, advanced technologies are employed to improve performance. For example, the motor cases are fabricated with a newly developed high-performance steel, and the second-stage and third-stage motors are equipped with deployable nozzles which give large expansion ratios.

For large payloads, the other space organization, NASDA, has the H-I rocket, which is currently operational, with a LEO capability of 3 tons. NASDA has also been developing a next generation launch vehicle, the $\mathrm{H}-\mathrm{II}$ rocket. The $\mathrm{H}$-II will be powered by two stages of liquid hydrogen engines and capable of launching 10 tons for LEO or a 2.2-ton geostationary satellite. The main features of H-I and H-II vehicles are also listed in Table 1. A possibility of occasional use of these vehicles of NASDA for scientific missions of ISAS is not excluded, whenever the need is fully justified. Thus, in addition to the regular programs with our own M-V vehicles, this possibility allows us to consider larger-scale cooperative missions in future. 